\title{
In reply: Reassessment of a meta-analysis of intraoperative cerebral-oximetry-based management studies
}

\author{
Andrés Zorrilla-Vaca, BSc (D) Ryan Healy, BSc
}

Received: 22 November 2018/Revised: 3 February 2019/Accepted: 13 April 2019/Published online: 26 August 2019

(c) Canadian Anesthesiologists' Society 2019

\section{To the Editor,}

In response to the letter by Davis and Saunders, ${ }^{1}$ we carefully reviewed our article published earlier in the Journal. $^{2}$ First, on re-review of our original paper, we noted that we had mislabelled Figs 5 and 6 leading Davis and Saunders to mistakenly note that six articles were analyzed for postoperative delirium (POD) as shown erroneously in Fig. 6. Importantly, we did correctly state that, "Four RCTs specifically assessed for postoperative delirium. There was no significant difference..." and if Figs 5 and 6 had been correctly labelled, it would have been clear that both figures correspond to data of red blood cell transfusions (six articles) and that none of the graphs presented data related to POD. Specifically, Fig. 5 represents the overall analysis for transfusions while Fig. 6 corresponds to a subgroup analysis of transfusion outcome. In re-reviewing our original submission, we now realize that the pooled risk ratio reported in Fig. 5 is not the same as that reported in the text (risk ratio, 0.90; $95 \%$ confidence interval [CI], 0.63 to $1.29 ; P=0.57$ ), which was very similar to the effect size noted in the reanalysis of Davis and Saunders. Nevertheless, we included the following four studies in the analysis of POD (according

Electronic supplementary material The online version of this article (https://doi.org/10.1007/s12630-019-01470-0) contains supplementary material, which is available to authorized users.

A. Zorrilla-Vaca, BSc ( $₫)$

School of Medicine, Universidad del Valle, Hospital

Universitario del Valle, Cali, Colombia

e-mail: andres.zorrilla@correounivalle.edu.co

R. Healy, BSc

Graduate Medical Sciences, Boston University School of Medicine, Boston, MA, USA to the table published in the original article) instead of only three articles (included by Davis and Saunders): Ballard et al. (non-published data provided by authors), ${ }^{3}$ Colak et al. ${ }^{4}$ Deschamps et al., ${ }^{5}$ and Lei et $a l .{ }^{6}$ We apologize for this error in Figs 5 and 6 of our original manuscript and emphasize that the values for POD reported in the text remain the correct ones. The correct figures can now be seen in the Electronic Supplementary Material (ESM).

Davis and Saunders also found inconsistences in the number of transfusions that we reported from the study by Colak et al. This was due to a misinterpretation on our part of the term "w/o" in their Table 2, which was not explained in the legends. We also did note the error of including the study by Cohn et al., which monitored oximetry on the thenar eminence rather than cerebral oximetry. Therefore, in the reanalysis we excluded Cohn et al. from the length of hospital stay outcome and found a similar non-significant result (standardized mean difference [SMD] $-0.07 ; 95 \% \mathrm{CI},-0.19$ to $0.05 ; P=0.26$ ).

David and Saunders also point to our changes from the registered PROSPERO protocol, that, importantly, had been registered before beginning our data search. Nevertheless, we do note that the analysis plan was subsequently modified while reading the included articles and writing the manuscript's methodology. Although not typically recommended, modifications to original protocols are often seen in meta-analyses. For example, the subgroup analysis by intervention algorithm was exploratory and not defined a priori in the protocol. We feel, however, that as long as otherwise adequate methods are used, these modifications should not be seen as a limitation. Furthermore, Davis and Saunders point out that they used mean differences for analyzing continuous variable data. Nevertheless, because of the extensive analyses of sample size and varying criteria for hospital discharge, we found 
SMD to be a better option. The SMD also provided an advantage in that the effect size could be interpreted as suggested by Cohen et al. (large SMD, > 0.8; medium SMD, 0.5-0.8; small SMD, 0.2-0.5; very small SMD, < $0.2)^{7}$

With regards to their concern about the Denault algorithm, it should be noted that the algorithm has multiple steps and it should be read in detail. Davis and Saunders grouped the studies that only used the major steps of the Denault algorithm (omitting specific steps). We disagree with their reassessment because the reanalysis combined the results of heterogeneous interventions, which is not recommended in a meta-analysis. Therefore we maintain that only seven studies followed the specific Denault algorithm in detail.

Lastly, despite our intention (as indicted in our PROSPERO registration) to do a subgroup analysis based on the devices used, this was not performed because most of the studies used the INVOS monitor. Thus, their statement that "outcomes might be influenced by interactions among devices" is not well supported.

In summary, we stand by the conclusions drawn from our meta-analysis and suggest that the "errors" called into question by Davis and Saunders were primarily caused by an error in Figs 5 and 6 that only present data of transfusions (see ESM for correct figures). In addition, we have verified the accuracy of the non-significant risk ratio for POD as reported in our original article. With regards to the data of transfusions, we apologize for the erroneous inclusion of the studies by Colak et al. and Cohn et al. and we agree with the reanalysis (see ESM). We disagree with the subgroup reanalysis by intervention algorithm and invite the readers to carefully analyze the specific interventions based on cerebral-oximetry monitoring. We recommend that future studies be directed towards assessing novel interventions.

Conflicts of interest None declared.

Editorial responsibility This submission was handled by Dr. Hilary P. Grocott, Editor-in-Chief, Canadian Journal of Anesthesia.

\section{References}

1. Davis JA, Saunders R. Reassessment of a meta-analysis of intraoperative cerebral oximetry-based management studies. Can J Anesth 2019; 66: this issue. DOI: https://doi.org/10.1007/s12630019-01469-7.

2. Zorrilla-Vaca A, Healy R, Grant MC, et al. Intraoperative cerebral oximetry-based management for optimizing perioperative outcomes: a meta-analysis of randomized controlled trials. Can J Anesth 2018; 65: 529-42.

3. Slater JP, Guarino T, Stack J, et al. Cerebral oxygen desaturation predicts cognitive decline and longer hospital stay after cardiac surgery. Ann Thorac Surg 2009; 87: 36-44.

4. Colak Z, Borojevic M, Bogovic A, Ivancan V, Biocina B, MajericKogler $V$. Influence of intraoperative cerebral oximetry monitoring on neurocognitive function after coronary artery bypass surgery: a randomized, prospective study. Eur J Cardiothorac Surg 2015; 47: 447-54.

5. Deschamps A, Lambert J, Couture P, et al. Reversal of decreases in cerebral saturation in high-risk cardiac surgery. J Cardiothorac Vasc Anesth 2013; 27: 1260-6.

6. Lei L, Katznelson R, Fedorko L, et al. Cerebral oximetry and postoperative delirium after cardiac surgery: a randomised, controlled trial. Anaesthesia 2017; 72: 1456-66.

7. Cummings $P$. Arguments for and against standardized mean differences (effect sizes). Arch Pediatr Adolesc Med 2011; 165 : 592-6.

Publisher's Note Springer Nature remains neutral with regard to jurisdictional claims in published maps and institutional affiliations. 